OPEN ACCESS

Edited by:

Rocío Martínez De Pablos,

University of Seville, Spain

Reviewed by:

Fabrizio Palitti,

Università degli Studi della Tuscia, Italy

Ray Truant,

McMaster University, Canada

*Correspondence:

Yanfeng Song

songyanfeng@|zu.edu.cn

Degui Wang

wangdegui@/zu.edu.cn

${ }^{\dagger}$ These authors have contributed

equally to this work

Specialty section:

This article was submitted to

Cellular Neuropathology,

a section of the journal

Frontiers in Cellular Neuroscience

Received: 10 April 2019 Accepted: 09 August 2019

Published: 23 August 2019

Citation:

Guo Z, Tian Y, Guo Y, Li B, Liu X,

Xie K, Song $Y$ and Wang D (2019)

RAD6B Plays a Critical Role

in Neuronal DNA Damage Response

to Resist Neurodegeneration.

Front. Cell. Neurosci. 13:392.

doi: 10.3389/fncel.2019.00392

\section{RAD6B Plays a Critical Role in Neuronal DNA Damage Response to Resist Neurodegeneration}

\author{
Zhao Guo't, Yingxia Tian²t, Yingli Guo', Boya Li', Xiangwen Liu', Kun Xie', \\ Yanfeng Song ${ }^{1 *}$ and Degui Wang ${ }^{1 *}$ \\ ' Department of Anatomy and Histology, School of Basic Medical Sciences, Lanzhou University, Lanzhou, China, \\ ${ }^{2}$ Department of Internal Medicine, Gansu Provincial Academic Institute for Medical Research, Lanzhou, China
}

RAD6 participates in DNA double-strand breaks (DSBs) repair by ubiquitinating histone $\mathrm{H} 2 \mathrm{~B}$ in mitotic cells. In terminally differentiated cells, however, the mechanisms of DNA damage repair are less well known. In this study, we investigate whether RAD6B is involved in DSBs repair in neurons and effects of RAD6B deficiency on neuronal survival. We compared neurons of RAD6B-deficient mice with those of littermate wild type (WT) mice and induced DNA damage by X-ray irradiation. We provide evidence that RAD6B is essential for neural DDR and RAD6B deficiency results in increased genomic instability and neurodegeneration. Moreover, higher levels of p53 and p21 are present in the brains of RAD6B-deficient mice, which may be responsible for neuronal senescence, and degeneration. In addition, behavioral experiments show that RAD6B-deficient mice exhibit marked learning and memory deficits. In conclusion, these findings suggest that RAD6B is critical for neural integrity and that the absence of RAD6B accelerates neurodegeneration in mice.

Keywords: RAD6B, aging, histone ubiquitination, DNA damage, neurodegeneration, senescence

\section{INTRODUCTION}

Biological cells are affected by various genotoxic stressors from endogenous and exogenous sources, and it is estimated that a typical mammalian cell undergoes $\sim 2 \times 10^{5}$ lesions per day (Harper and Elledge, 2007; Ouyang et al., 2015; White and Vijg, 2016; Hegde et al., 2017). DNA DSBs are the most harmful to cells (Jackson and Bartek, 2009; Ouyang et al., 2015), which often lead to chromosomal rearrangements, senescence, tumorigenesis, or cell death (Lee and Mckinnon, 2007; White and Vijg, 2016). To avert those potentially devastating consequences, cells initiate highly evolved DNA damage response (DDR), and detect and repair damaged DNA. DSBs always occur when cells are exposed to genotoxic agents such as ionizing radiation and oxidative stress and are detected by two sensor proteins, the MRE11/RAD50/NBS1 (MRN) complex and the Ku70/Ku80 heterodimer (White and Vijg, 2016). Then ATM is activated and phosphorylates histone variant $\mathrm{H} 2 \mathrm{AX}$ to form $\gamma-\mathrm{H} 2 \mathrm{AX}$ and bind to the break sites. $\gamma-\mathrm{H} 2 \mathrm{AX}$ also forms a binding site for MDC1, which recruits additional downstream repair factors, such as RNF8, 53BP1 and BRCA1, to gather at the sites and repair these breaks (Harper and Elledge, 2007; Marteijn et al., 2009; Liu et al., 2013; Ouyang et al., 2015). Based on different factors, including the cell type, cell-cycle stage and the severity of the damage, accurate repair leads to restoration of an intact double helix, while failed repair often cause apoptosis, and senescence or even tumor formation (Barzilai, 2010). 
Protein ubiquitination is a series of complex cascade reactions including three enzymes: ubiquitin activating enzymes (E1s), ubiquitin conjugating enzymes (E2s), and ubiquitin ligases (E3s) (Walden et al., 2003; Chen et al., 2012). Along with other modifications such as methylation, acetylation and phosphorylation, ubiquitination plays important roles in many cellular processes (Kim et al., 2009), including the regulation of cell cycle, apoptosis, gene expression, transcription regulation, DNA damage repair, and protein degradation (Ciechanover, 1994; Biochem, 1998; Varshavsky, 2005; Chen et al., 2012). RAD6 is first identified as an important E2 in yeast and interacts with three separate E3s (Ubr1, Rad18, and Bre1) to participate in DDR. However, there are still some problems to be clarified for RAD6-mediated DNA repair involving its function with Brel in mono-ubiquitinating the histone $\mathrm{H} 2 \mathrm{~B}$ residue lysine 123 . RAD6 mutation causes yeasts to be highly sensitive to ultraviolet light (Game and Chernikova, 2009; Chen et al., 2012). In addition, Saccharomyces mutants deficient in $\mathrm{H} 2 \mathrm{~B}$ mono-ubiquitination also shown irradiation-sensitive and abortive DNA damage repair, gene silencing, and cellcycle checkpoints (Game and Chernikova, 2009; Chernikova et al., 2012). RAD6A and RAD6B are two homologs of yeast RAD6 and mice are non-viable if both homologs are absent (Chen et al., 2012).

While in the central nervous system, protected by the skull and blood-brain barrier, the most common threat to the neuronal genome comes from metabolic reactive oxygen species (ROS), which cause oxidative DNA damage, including single strand breaks (SSBs) (Madabhushi et al., 2014). However, under certain conditions, SSBs may be converted to DSBs, such as two SSBs that are in close proximity to each other (Madabhushi et al., 2014). And recent studies have shown that DNA DSBs are also formed in normal physiological/metabolic processes. On the other hand, the ability of neurons to repair damage decreases with aging. Thus, appropriate responses to DNA DSBs and accurate repair of broken DNA are required to maintain homeostasis and organismic survival (Lee and Mckinnon, 2007).

In recent years, there has been a continuous stream of evidences that mutations of DNA repair genes frequently cause severe defects in the central nervous system (Herrup et al., 2013) and that DNA damage is not only correlated with the aging process but also proposed to accelerate it (Barzilai and Mckinnon, 2013; Canugovi et al., 2013; Hegde et al., 2017). Furthermore, several neurodegenerative diseases, such as ataxia-telangiectasia (A-T), Alzheimer's disease (AD), and Parkinson's disease (PD), are closely related to DNA damage repair (Jackson and Bartek, 2009; Reynolds and Stewart, 2013). Our latest experiments have shown that loss of the ubiquitin ligase RNF8 leads to neurodegeneration in mice and DNA damage preceding dopamine neuron degeneration in PD mice (Ouyang et al., 2015; Wang et al., 2016). Bioinformatics analysis also indicates that $293 \mathrm{~T}$ cells with RAD6B deficiency express higher levels of mRNA involving neurodegeneration. To further explore the effect of RAD6B deficiency on neurons, we focus on the function of RAD6B in neural DDR and phenotypes of neurons in RAD6Bdeficient mice.

\section{RESULTS}

\section{RAD6B Is Essential for Neural DNA DSBs Repair}

To compare the repair processes for DSBs in the neurons of RAD6B-deficient mice with those in the neurons of WT mice, we observed the formation of ionizing radiation-induced nuclear foci (IRIF) at the broken sites after inducing damage by X-ray irradiation. $\gamma$-H2AX foci were used as a marker of DNA damage, while MDC1, 53BP1, RNF8, and BRCA1 were co-stained with $\gamma$-H2AX as repair factors. As shown in Figure 1, there were numerous 53BP1, BRCA1, RNF8, and MDC1 foci in the neurons of WT mice. In addition, most of them colocalized with the corresponding $\gamma$-H2AX foci, indicating that they were recruited to the broken sites after X-ray irradiation. By contrast, 53BP1 and BRCA1 foci were decreased dramatically in the neurons of RAD6B-deficient mice after X-ray irradiation, suggesting that RAD6B deficiency caused them to be recruited less to the break sites. However, in the neurons of RAD6B-deficient mice, almost the same proportions of MDC1 and RNF8 foci of were detected as in the neurons of WT mice, respectively (Figures 1A,B). These results suggest that $\mathrm{RAD} 6 \mathrm{~B}$ is involved in the repair process for DNA DSBs in neurons. Deficiency of RAD6B leads to incomplete recruitment of downstream repair factors, including 53BP1 and BRCA1, but the upstream proteins MDC1 and RNF8 are not affected.

\section{Loss of RAD6B Leads to Decreased H2B Ubiquitination}

Post-translational modifications (PTMs) of histones are important regulators of the structure of chromosomes. We next focused on the substrate histones of RAD6B ubiquitination in neurons and measured the ubiquitination levels of the histones $\mathrm{H} 2 \mathrm{~A}$ and $\mathrm{H} 2 \mathrm{~B}$. As shown in Figures 2A,B, knockout of RAD6B leads to significant reduction in the ubiquitination of $\mathrm{H} 2 \mathrm{~B}$ compared with the WT mouse brains. No evident increase was detected in the expression of ubH2B in RAD6Bdeficient mouse brains, while $\mathrm{ubH} 2 \mathrm{~B}$ in the WT mouse brains increased obviously after X-ray irradiation. However, ubH2A in the brains of WT mice and RAD6B-deficient mice both increased after IR, and higher level of $\mathrm{ubH} 2 \mathrm{~A}$ was observed in the brains of RAD6B-deficient mice. The relative expression levels of $\mathrm{H} 2 \mathrm{~A}$ and $\mathrm{H} 2 \mathrm{~B}$ did not show clear changes between groups (Figures 2A,B). Consistent with ubH2B, the level of H3K79me2 in RAD6B-deficient mouse brains decreased significantly (Figures 2C,D).

\section{RAD6B Deficiency Leads to Increased Genomic Instability and Neurodegeneration}

While knocking down RAD6B did not significantly influence cell proliferation compared with siNeg treated cells within $72 \mathrm{~h}$, cell growth was obviously inhibited with $2 \mathrm{~Gy}$ of X-ray treatment (Figure 3A). To determine the effect of RAD6B deficiency on neuronal genomic integrity, we evaluated the 


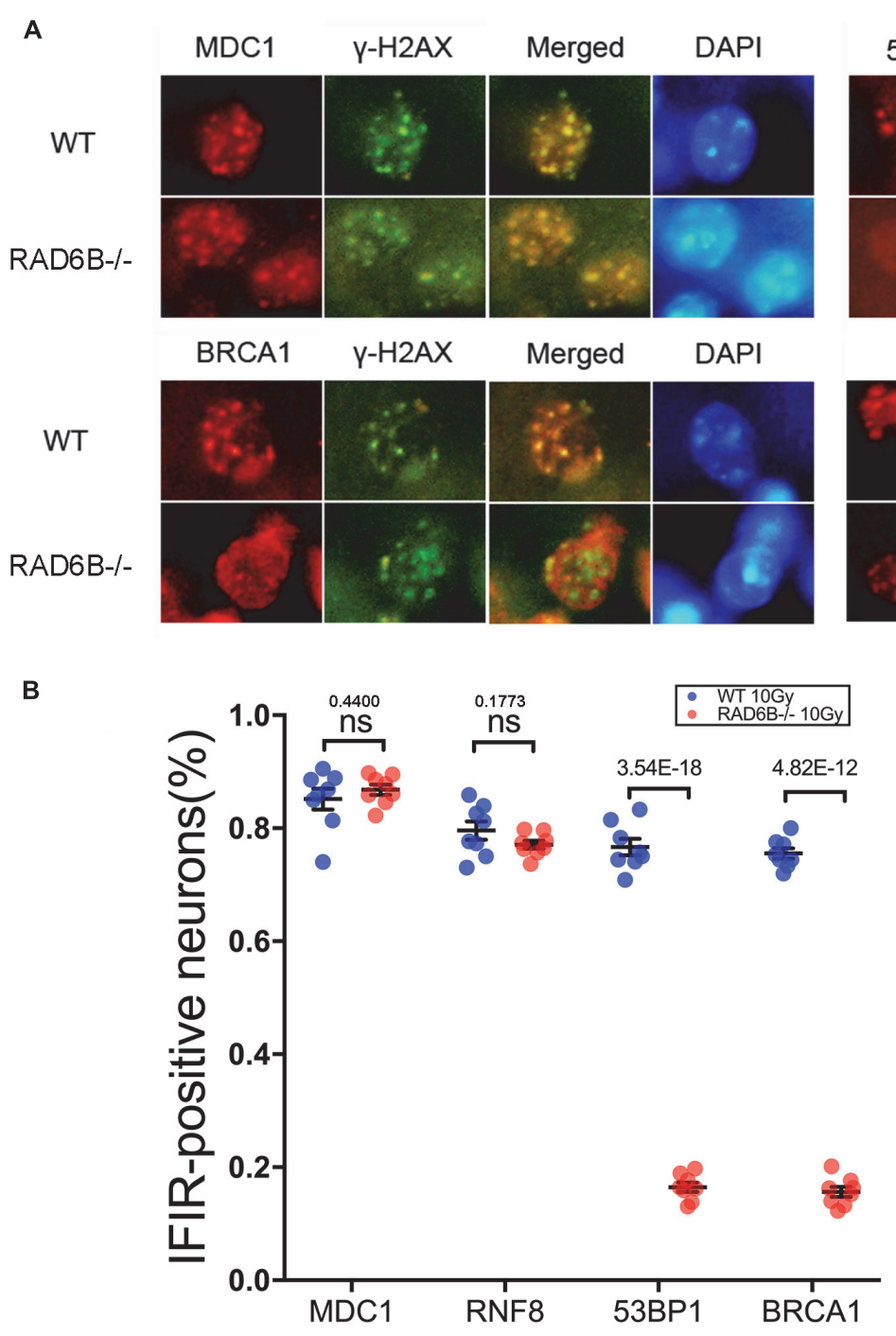

FIGURE 1 | RAD6B is involved in DNA damage repair in neurons after IR. (A) RAD6B-deficient mice and control mice were simultaneously exposed to X-rays at a dose of 10 Gy. After $2 \mathrm{~h}$ of recovery, frozen sections of brain tissues were obtained and immunofluorescence was used to detect the formation of IRIF. Anti-MDC1, anti-53BP1, anti-BRCA1, and anti-RNF8 antibodies were applied separately for co-immunostaining with anti- $\gamma$-H2AX antibody. Scale bar $=20 \mu \mathrm{m}$. (B) The average percentages of IRIF-positive neurons were analyzed and are displayed with scatter diagrams. All values are presented as the mean \pm SEM ( $n=8$ ). Student's $t$-test.

formation of MN. A total of 1000 binucleated cells in six separate experiments for each group were scored to evaluate the frequency of $\mathrm{MN}$ induction and cells contained at least one MN were counted as MN-positive. Shin Koyama reported that there was a threshold of dose of X-rays for $\mathrm{MN}$ induction and no significant difference at doses lower than 0.05Gy was detected compared with the control. Interestingly, a small amount of $\mathrm{MN}$ was also detected in siRAD6B-treated RPE1 cells without X-ray irradiation. However, in the case of X-ray treatment, a large amount of MN was produced, about four folds as much as that of siNeg treated cells (Figures 3B,C). Generally, MN originate from chromosome fragments or whole chromosomes lagging behind during anaphase of mitosis. Therefore, these results suggest that RAD6B deficiency causes increase of genomic instability in neurons due to the defect of DSBs repair.
Analysis of the nuclei and neurites in neurons of the RAD6Bdeficient mice with silver staining revealed that more nuclei in RAD6B-deficient mice became irregular and deeply stained, while neurons in the WT mice had relatively regular morphology and included only a few deeply stained cells. X-ray irradiation increased the staining of some nuclei, but the neurons in RAD6B-deficient mice were more pronounced (Figures 3D,E). More degenerated neurites (tangles, thickening, and deeper staining) were also observed in the brains of RAD6B-deficient mice (Supplementary Figure S2C).

Astrocytes, as the most important supporting cells in the central nervous system, become larger and grow longer protrusions with more branches when activated. Furthermore, upregulation of glial fibrillary acidic protein (GFAP) in activated astrocytes indicates neuronal injury and degeneration. We 
A

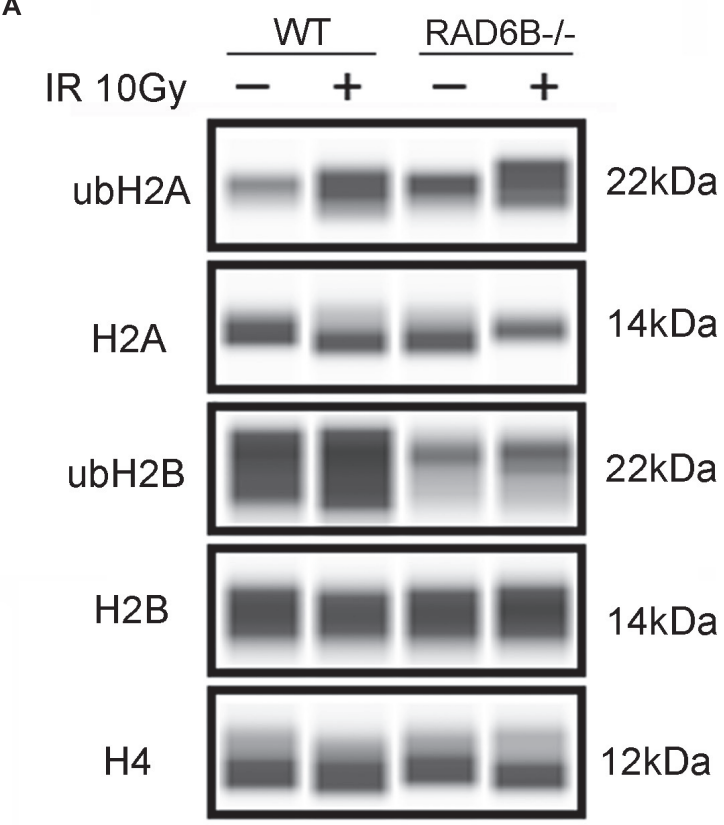

C

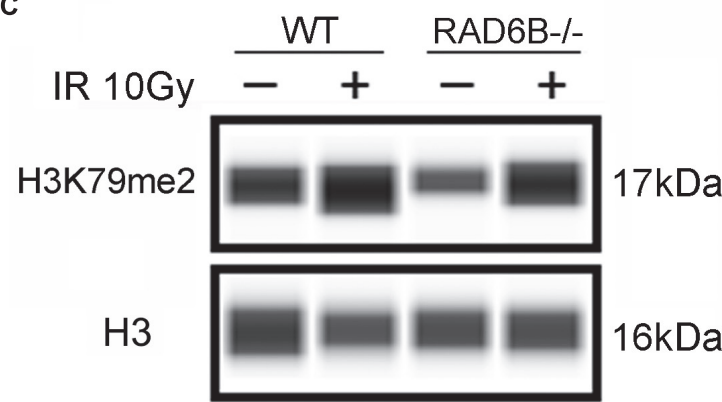

B

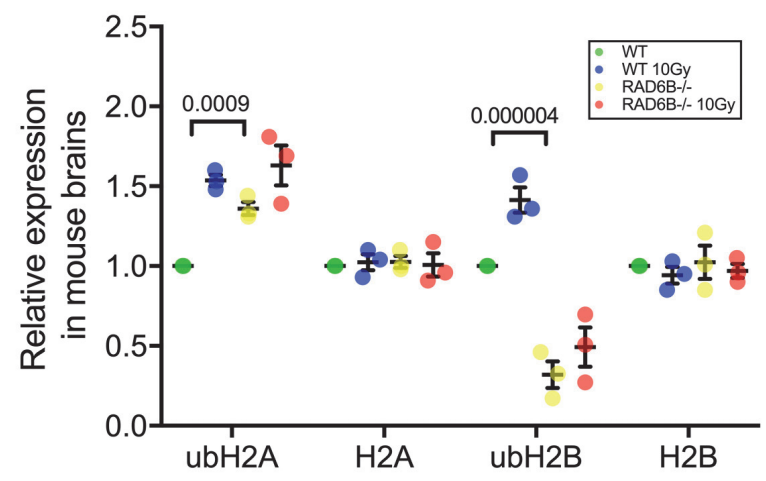

D

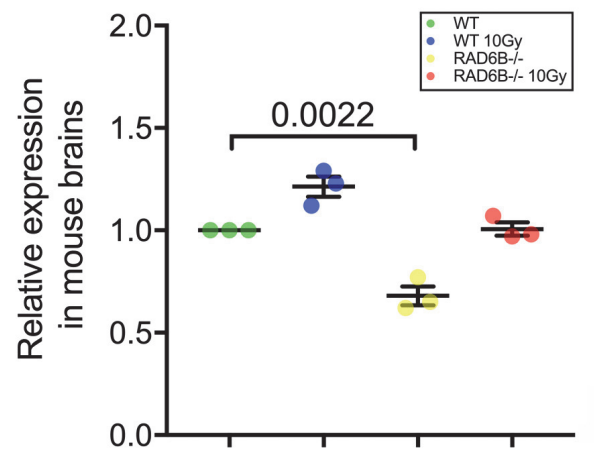

FIGURE 2 | Loss of RAD6B causes decreased H2B ubiquitination. (A) RAD6B-deficient mice and control mice were treated with 10 Gy of X-rays or not. After recovery, histones were extracted from the brain in diluted hydrochloric acid, and the relative levels of ub-H2A, ub-H2B, $\mathrm{H} 2 \mathrm{~A}, \mathrm{H} 2 \mathrm{~B}$, and $\mathrm{H} 4$ were detected through western blotting. (B) The gray values of each group were measured, and the relative expression is displayed with a scatter diagram. All values are presented as the mean \pm SEM $(n=3)$. Student's $t$-test. (C) The relative levels of dimethylation of histone H3K79 were detected through western blotting. (D) The scatter diagram showing the relative expression of dimethylation of histone H3K79. All values are presented as the mean \pm SEM $(n=3)$. Student's $t$-test.

measured GFAP and found that there were more astrocytes activated in RAD6B-deficient mice than in WT mice. X-ray irradiation also increased the activation of astrocytes in the brains of RAD6B-deficient mice and WT mice, and RAD6Bdeficient mice showed significantly more astrocytic activation after X-ray irradiation (Figures 3F,G). In addition, there were more degenerated neurons surrounded by microglial cells in RAD6B-deficient mice (Supplementary Figures S2D,E).

Analysis of immunohistochemistry with anti-NeuN revealed that the number of neurons in the RAD6B-deficient mice was significantly reduced compared with homologous 12-monthold WT mice; the RAD6B mutants had approximately $3 / 4$ as many neurons as the WT mice. In addition, RAD6B-deficient mice lost more neurons than WT mice after X-ray irradiation (Supplementary Figures S2A,B). We further performed CV staining to investigate neuronal loss in those knockout mice over time. The loss of pyramidal neurons resulting from RAD6B deficiency gradually increased, and those mice lost more neurons than homologous WT mice from 6 to 12 months of age (Figures 3H,I). Above all, changes in brain slices demonstrate that RAD6B deficiency results in a reduction in the number of neurons.

Argyrophilic grain disease, characterized by the presence of argyrophilic grains, is a progressive degenerative disorder of neurons and becomes increasingly prevalent with advancing age (Braak and Braak, 1998; Yoshida et al., 2017). Unexpectedly, we found a large number of argyrophilic grains (AGs) in the hippocampus and dentate gyrus in 9-month-old RAD6Bdeficient mice (Figures 4A,B). Moreover, there was no significant change in the number of AGs after X-ray irradiation. After a further study of age-related changes in AGs, we noticed that numerous $A G$ deposits were present in the hippocampus and 
A

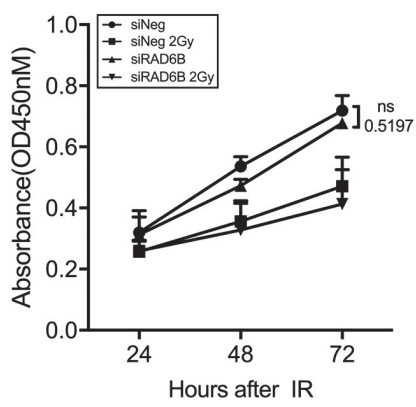

D

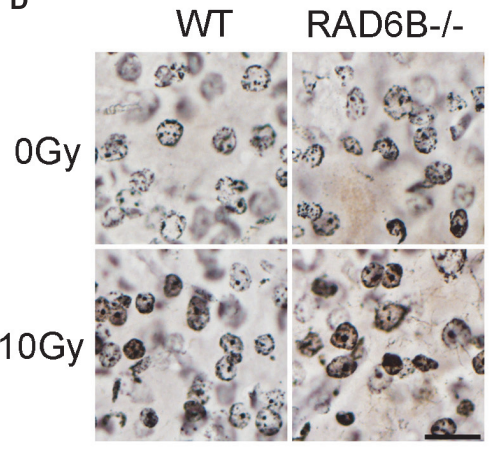

B

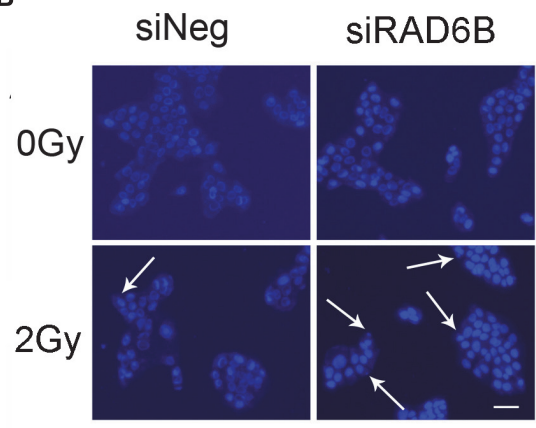

C

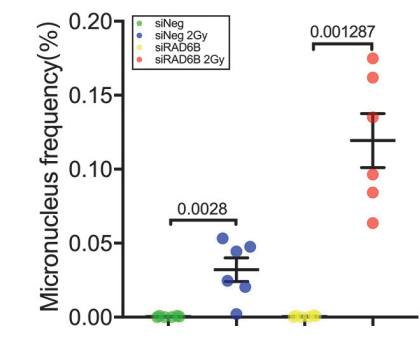

E

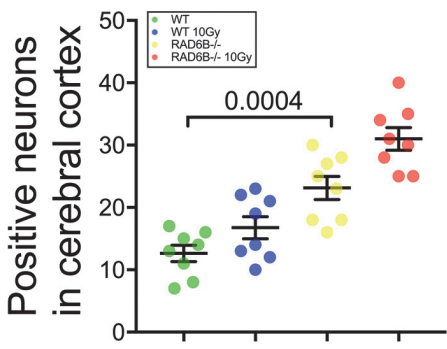

H

WT 0Gy WT 10Gy RAD6B-I-0Gy RAD6B-I-10Gy

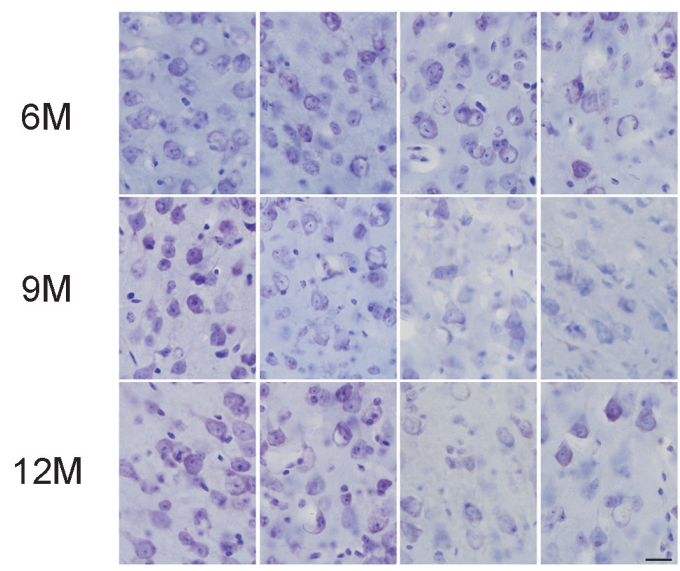

G

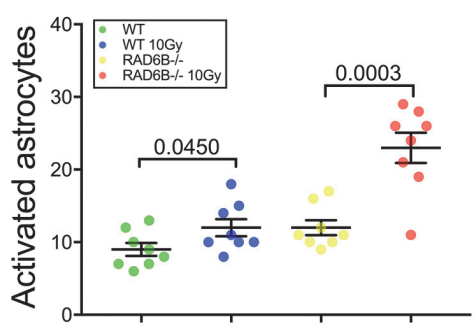

1

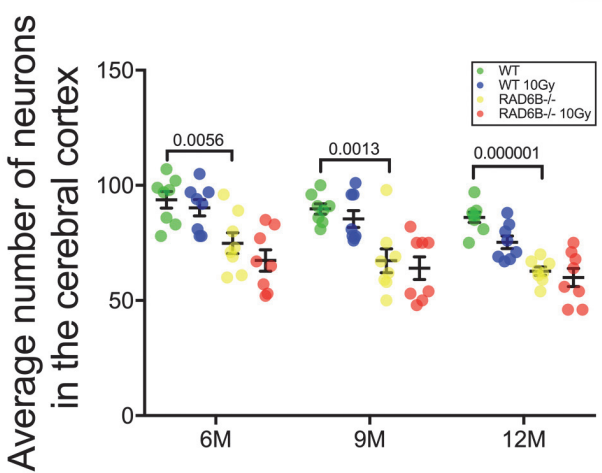

FIGURE 3 | Deficiency of RAD6B leads to neurodegeneration and loss of neurons. (A) Proliferation of siNeg or siRAD6B-treated RPE1 cells after 2Gy of X-ray irradiation or not. All values are presented as the mean \pm SEM $(n=3)$. Two-way ANOVA. (B) Photomicrographs of siNeg or siRAD6B-treated RPE1 cells scored in the MN test, the arrow indicating the representative MN. Scale bar $=50 \mu \mathrm{m}$. (C) The frequency of MN formation in RPE1 cells, the data are presented as means \pm SEM $(n=6)$. Student's $t$-test. (D) Plot of neurons in silver-stained slices from 12-month-old mice. Scale bar $=20 \mu \mathrm{m}$. (E) Scatter diagram displaying the average density of abnormal neurons in the cerebral cortex of 12-month-old mice. All values are presented as the mean \pm SEM $(n=8)$. Student's $t$-test. (F) Two hours after X-ray irradiation, the brain slices of 6-month-old mice underwent immunofluorescence staining of GFAP to show astrocytes. Scale bar $=20 \mu \mathrm{m}$. (G) A scatter diagram showing the relative activation of astrocytes in the brains of mice in each group after $2 \mathrm{~h}$ of recovery. All values are presented as the mean $\pm \mathrm{SEM}$ $(n=8)$. Student's $t$-test. $(\mathbf{H}) \mathrm{CV}$ staining of slices from mice of different ages; the mice were treated with $\mathrm{X}$-rays and allowed to recover for days or were not treated with X-rays. Scale bar $=20 \mu \mathrm{m}$. (I) Scatter diagrams indicating the average number of neurons in each group at different ages. All values are presented as the mean \pm SEM $(n=8)$. Student's $t$-test. 
dentate gyrus in 6-month-old RAD6B-deficient mice. However, there were only sporadic deposits in brain slices from 12-monthold WT mice. RAD6B-deficient mice had more AG aggregates than their WT littermates at different ages (Figures 4C-E). These results further suggest that RAD6B deficiency accelerates neuronal senescence.

\section{RAD6B Deficiency Results in Neuronal Senescence}

DNA damage repair defects lead to accumulation of damages, resulting in apoptosis and senescence, which are widely accepted as the major mechanisms inhibiting tumor formation mediated by p53 (Campisi, 2005; Li et al., 2012). To evaluate the effects of RAD6B deficiency on neuron survival, we compared the senescence of neurons in the four groups of mice. Senescent cells are usually larger in size and express $\beta$-galactosidase with high enzyme activity at $\mathrm{pH}$ 6.0. The senescent neurons in the RAD6B-deficient mice were slightly more numerous than those in the WT mice, and greater numbers of senescent neurons were observed after X-ray irradiation in 12-month-old mice (Figures 5A,B). Similarly, RAD6B silencing also causes RPE1 cells to show increased activity of SA- $\beta$-gal as more senescent cells were detected compared to control cells 7 days after X-ray treatment (Figures 5C,D). Next, we compared the levels of activated caspase- 3 and mature form of AIF in WT mice and RAD6B-deficient mice to assess apoptosis, but no significant difference was detected (Figures 5E,F). These results suggest that inhibition of RAD6B induces defects in DNA damage repair and that neurons are more susceptible to senescence.

\section{Activation of P53 and P21 Contributes to the Senescence of RAD6B Deficient Neurons}

Failure of DNA damage repair leads to senescence, usually through two signaling pathways: the p53-p21 pathway and the p16-Rb pathway (Sherr and McCormick, 2002; Campisi, 2005; Cao and Li, 2015; Gil and Withers, 2016). To investigate the causes of neuronal senescence in RAD6B-deficient mice, we examined key factors in these two pathways. As shown in Figures 5G,H, the levels of p21 increased in both groups after X-ray irradiation. Meanwhile, in the absence of X-ray irradiation, the levels of p21 in RAD6B-deficient mice also increased significantly. In addition, changes in the expression of p53 protein were similar to the changes in p21. However, p53 had relative markedly higher expression than p21 in RAD6B-deficient mouse brains. The levels of p16 did not vary obviously between groups (Figures $\mathbf{5 G}, \mathbf{H}$ ). Those results suggest that deficiency of RAD6B leads to increased expression of p53 and p21, which may contribute to neural senescence in RAD6B-deficient mice.

\section{RAD6B Deficient Mice Exhibit Learning and Memory Impairment}

As the command center of the nervous system, the brain controls a number of functions, including learning and memory, motor control, homeostasis, information processing, and perception (Rulten and Caldecott, 2013). Interesting, we noticed that the learning and memory abilities of RAD6B-deficient mice were significantly lower than those of WT mice. To identify whether these changes were caused by RAD6B systemic knockout, we used RAD6B neuron-conditional knockout mice (ncKO) and their WT littermate controls to evaluate the differences in motor performance and learning and memory through avoidance experiments and Morris water maze. RAD6B ncKO mice looked more inactive and exhibited slow behavior and decreased movement, and the two genotypes showed apparent difference in motor ability (Figures 6A,B). In addition, RAD6B ncKO mice were smaller in size than the WT mice and weighed significantly less (Supplementary Figures S1C,D).

We subjected both genotypes to a spatial exploration experiment to evaluate their spatial learning and memory ability in the Morris water maze. The WT mice were more likely to enter the target region to find the escape platform, with shorter latency to enter the target region, a longer total duration of time spent there, and more entries into the region (Figures 6C-E).

In the passive avoidance response test, RAD6B ncKO mice made twice as many errors as WT mice (Figure 6F). In addition, their error latency was significantly shorter than that of WT mice (Figure 6G). These results indicate that the learning and memory abilities of RAD6B-deficient mice are lower than those of WT mice during the early learning process.

\section{DISCUSSION}

Maintaining genetic stability, especially in the central nervous system, is vital to individual survival and reproduction. Most neurons in human beings are developed within 3 years and survive for the individual's lifetime (Hegde et al., 2017). As DNA DSBs usually threaten cell survival, eukaryotic organisms will mainly repair the injury in two conservative ways: homologous recombination (HR) and non-homologous end joining (NHEJ) (Escribano-Díaz et al., 2013; Iyama, 2013). NHEJ can occur at any stage of the cell cycle, although it is not as precise as HR (Iyama, 2013; Rulten and Caldecott, 2013; Ouyang et al., 2015). Since neurons are terminally differentiated cells and cannot re-enter the cell cycle, there is no template for homologous recombination repair. NHEJ is the primary mechanism involved in neuronal DDR 4-6 h after X-ray irradiation and repairs 80$85 \%$ of DNA DSBs (Löbrich et al., 2010; Jeggo et al., 2011; Reynolds and Stewart, 2013; Ouyang et al., 2015). However, the mechanisms underlying this repair are less well known in terminally differentiated cells.

In response to DNA DSBs, the MRN complex and $\mathrm{KU}$ proteins will sense this fragmentation damage and rapidly recruit and activate downstream ATM and DNA-PKcs, respectively (Assenmacher and Hopfner, 2004; Jackson, 2005; Bartkova et al., 2008). ATM is phosphorylated at Ser1981 and binds to the break sites, where it assembles the histone variant $\mathrm{H} 2 \mathrm{AX}$ and phosphorylates it at Ser139 (Lavin, 2007; Marteijn et al., 2009; Rulten and Caldecott, 2013). Subsequently, MDC1 recognizes and binds to $\gamma$-H2AX via the BRCT domains and recruits downstream two important cell cycle checkpoint mediators: 53BP1 and BRCA1, both of which are also involved in many 
A

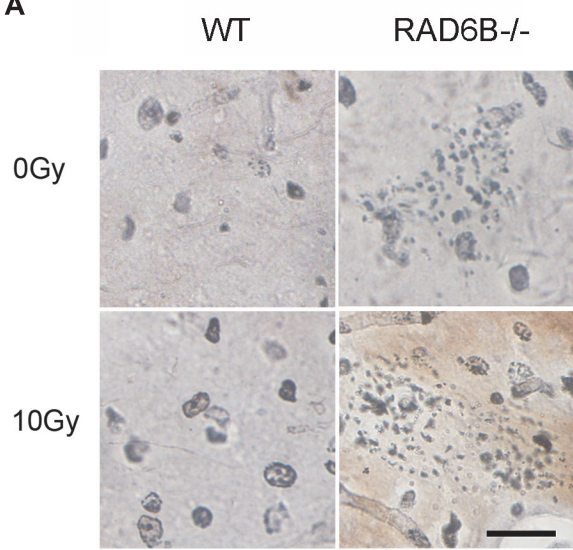

B

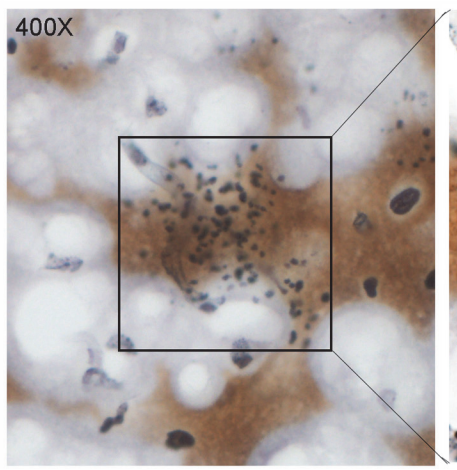

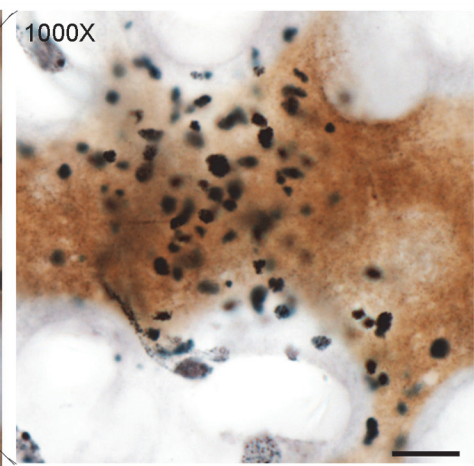

C

$6 \mathrm{M}$

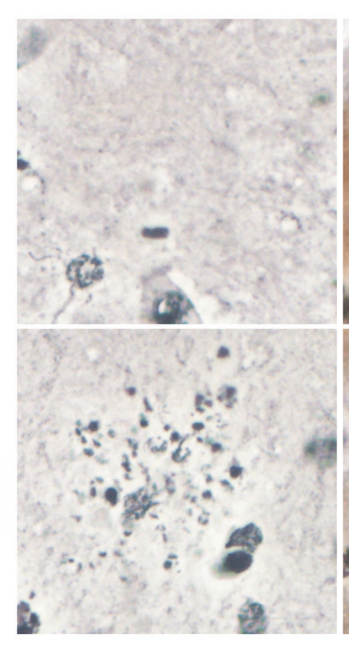

$9 \mathrm{M}$

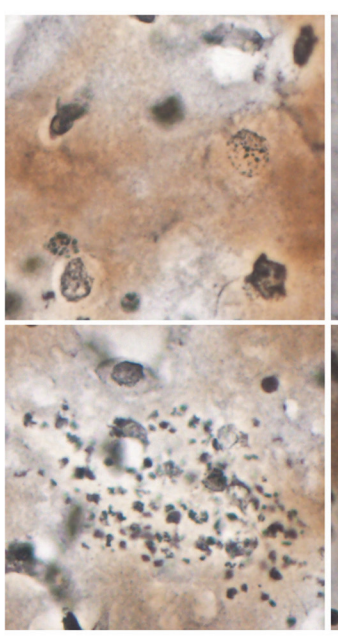

$12 \mathrm{M}$

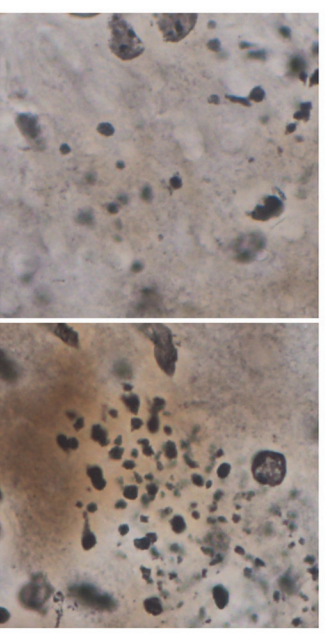

\section{$24 \mathrm{M}$}

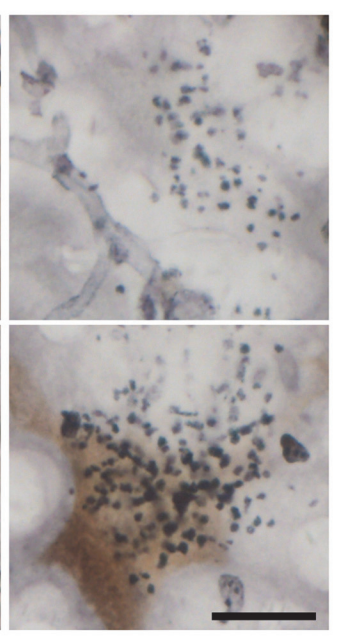

D

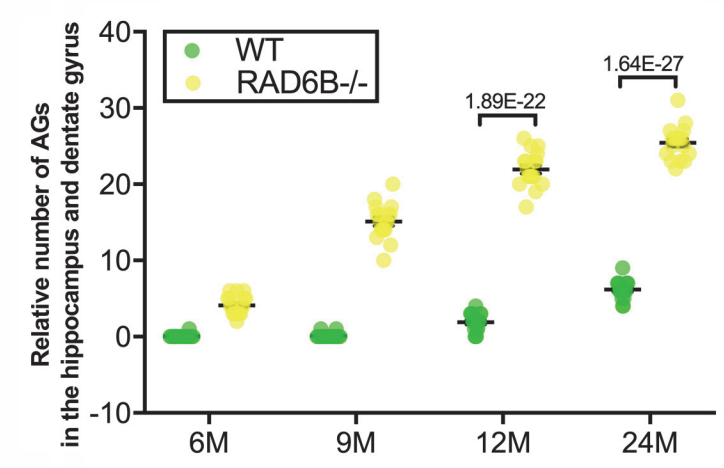

E

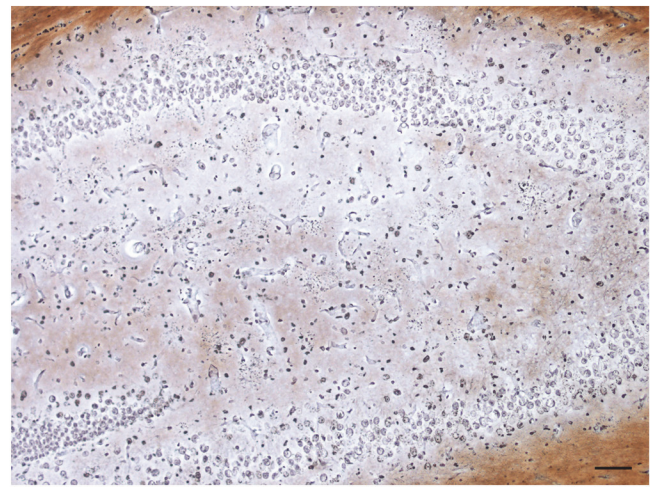

FIGURE 4 | RAD6B deficiency leads to abnormal accumulation of argyrophilic grains (AGs) in the hippocampus and dentate gyrus. (A) Brain slices were stained with silver, showing abnormal accumulation of AGs in the hippocampus. RAD6B-deficient mice and control mice were treated with 10 Gy of X-rays or left untreated. Scale bar $=20 \mu \mathrm{m}$. (B) A representative image shows the abnormal accumulation of AGs in the hippocampus and a magnified detail of the image. (C) Aggregation of AGs in hippocampus of WT mice and RAD6B-deficient mice at different ages. Scale bar $=10 \mu \mathrm{m}$. (D) The scatter diagram shows the relative number of AGs abnormally aggregated in the hippocampus of mice of different ages. All values are presented as the mean \pm SEM ( $n=18$ ). Student's $t$-test. (E) The silver-stained brain slices show that AGs are widely distributed in the hippocampus and dentate gyrus. Scale bar $=100 \mu \mathrm{m}$. 


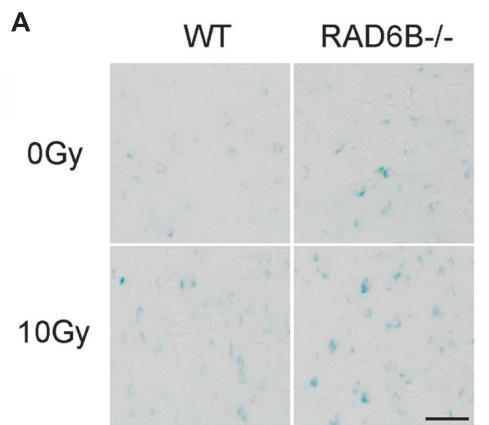

C

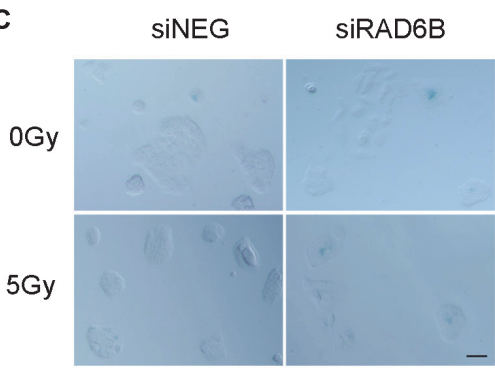

G

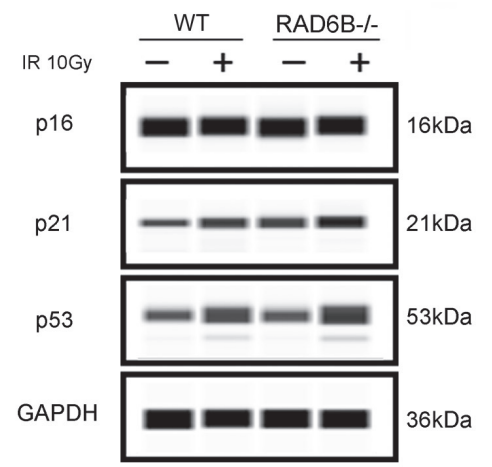

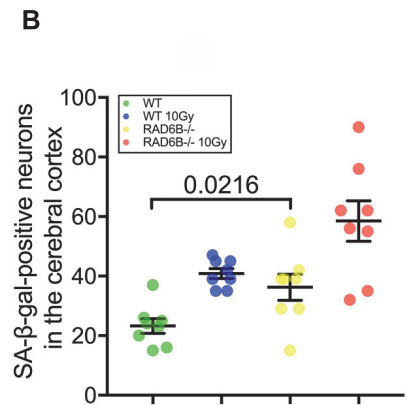

D

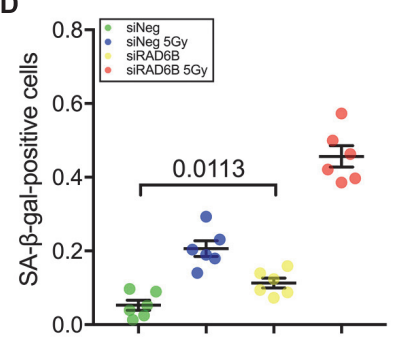

E

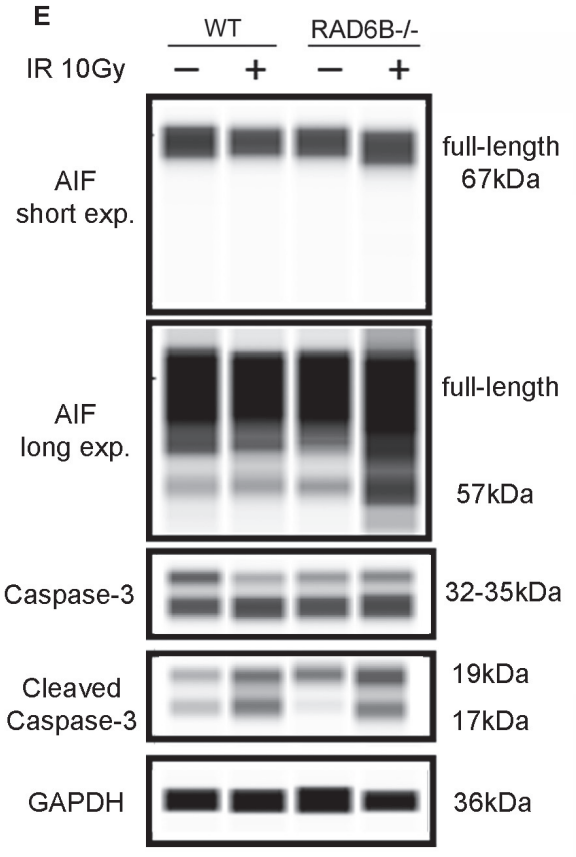

H

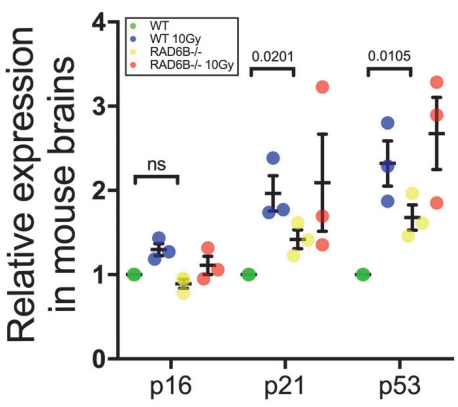

$\mathbf{F}$
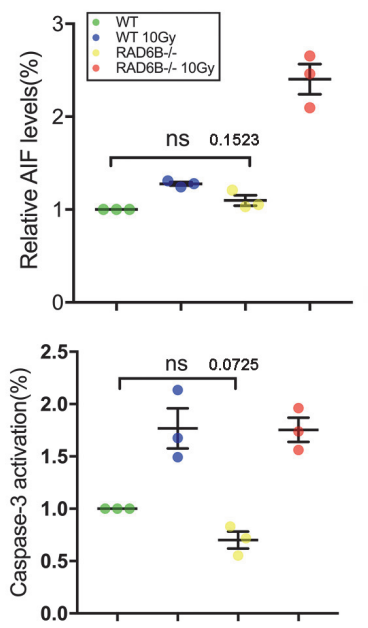

FIGURE 5 | RAD6B deficiency leads to increased neuronal senescence. (A) $\beta$-Galactosidase staining of slices from 12-month-old mice; the mice were either treated with X-rays and allowed to recover for 10 days or not treated with X-rays. Scale bar $=20 \mu \mathrm{m}$. (B) The scatter diagram showing the average number of senescent neurons in the cortex. All values are presented as the mean \pm SEM $(n=8)$. Student's $t$-test. (C) Photomicrographs of $\beta$-Galactosidase staining of siNeg or siRAD6B-treated RPE1 cells. Scale bar $=50 \mu \mathrm{m}$. (D) The bar chart displaying the percentage of senescent RPE1 cells, the data are presented as means \pm SEM $(n=6)$. Student's $t$-test. (E) The representative blot presenting caspase-3 and AIF levels analyzed through simple western. (F) A scatter diagram displaying the relative levels of cleaved caspase- 3 and mature form of AIF $(57 \mathrm{kDa})$ in each group. All values are presented as the mean \pm SEM $(n=3)$. Student's $t$-test.

(G) RAD6B-deficient mice and control mice, treated with $10 \mathrm{~Gy}$ of X-rays, or not. After a $2 \mathrm{~h}$ recovery period, the total protein of the brain was extracted, and a western blot was performed with the following primary antibodies: p16, p53, p21, and GAPDH. (H) The gray values of each group were measured, and the scatter diagram indicates the relative levels of these proteins. All values are presented as the mean $\pm \operatorname{SEM}(n=3)$. Student's $t$-test.

aspects of DNA repair. While 53BP1 mainly acts through its effector Rif1 to promote NHEJ, BRCA1 facilitates homologous recombination after resection of the broken ends (Bunting et al., 2010; Escribano-Díaz et al., 2013). Interestingly, deficiency of RAD6B caused mice to form fewer 53BP1 and BRCA1 foci in neurons with X-ray treatment, while the underlying mechanism needs further study. Previous studies reported that MDC1 recruits RNF8 at damage sites through the interaction between RNF8 and UBC13, which would ubiquitinate $\mathrm{H} 1$ and initiate subsequent ubiquitination events, including ubiquitylation of H2A-type histones mediated by RNF168 (Marteijn et al., 2009; Fradet-Turcotte et al., 2013; Thorslund et al., 2015; Mandemaker et al., 2017). BRE1 facilitates mono-ubiquitination of $\mathrm{H} 2 \mathrm{~B}$ by RAD6 through its ring finger domain. In addition, $\mathrm{ubH} 2 \mathrm{~B}$ is 
A

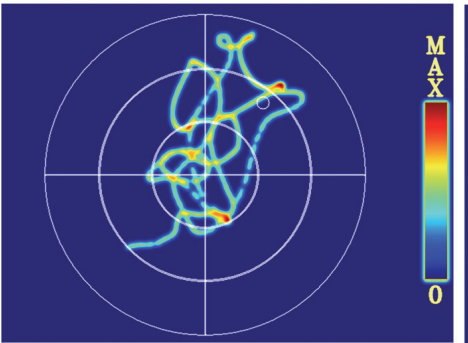

WT

C

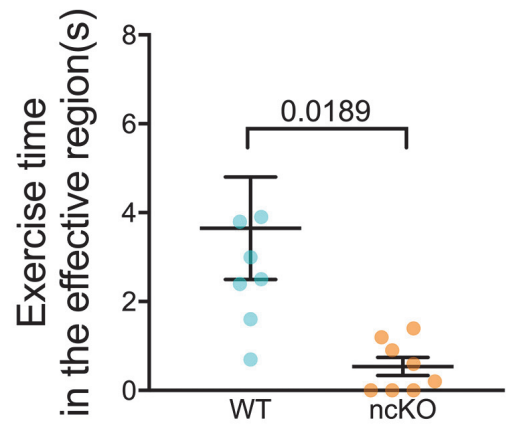

F

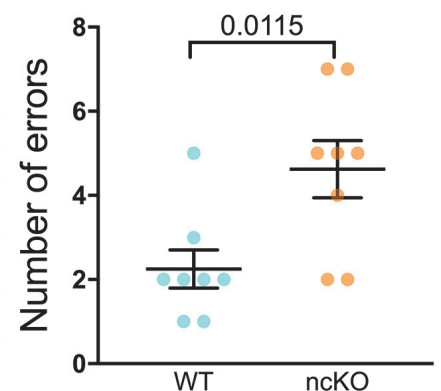

B

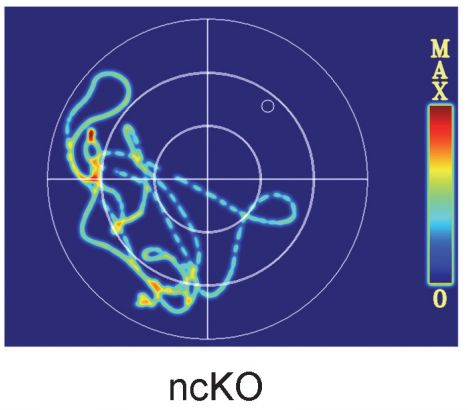

D

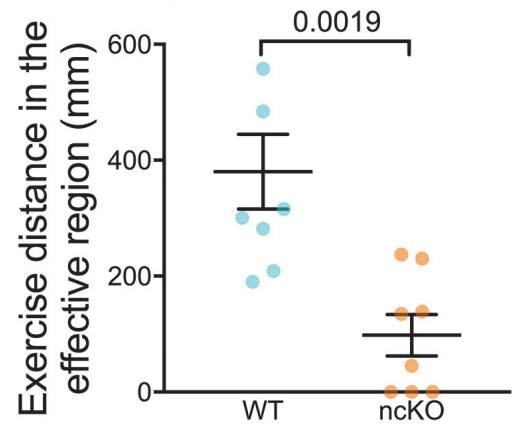

G

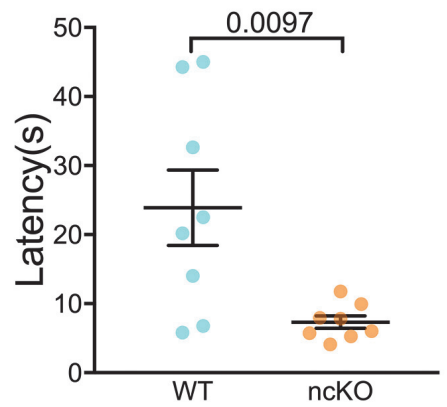

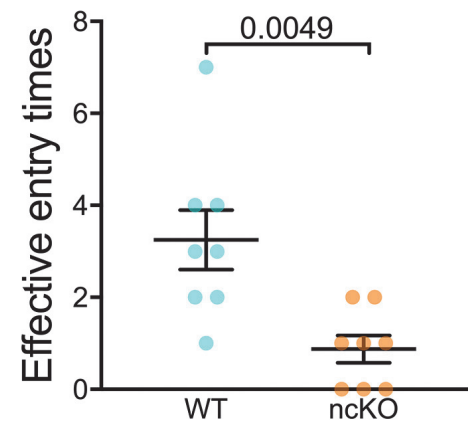

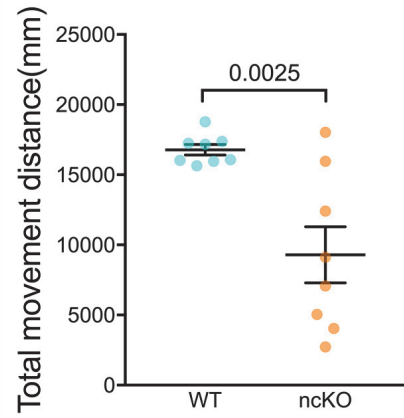

E

FIGURE 6 | RAD6B-deficient mice exhibit weight loss accompanied by learning and memory deficits. (A) Motion paths of RAD6B ncKO mice and normal mice in the Morris water maze (B-E). The scatter diagrams show total movement distance, time and distance in the target region and the frequency with which the two genotypes entered the target region in the Morris water maze. All values are presented as the mean \pm SEM $(n=8)$. Student's $t$-test. (F, $\mathbf{G})$ The number of errors and the latency of the first error on the passive avoidance response test are summarized and displayed in scatter diagrams. All values are presented as the mean \pm SEM $(n=8)$. Student's $t$-test

essential for the dimethylation and trimethylation of histones H3K4 and H3K79 (Ng et al., 2002; Kim et al., 2009; Chen et al., 2012; Kleiner et al., 2015; Van Oss et al., 2016), which are required for activation of chromatin, remodeling, and activation of transcription of corresponding genes. Our results demonstrated that the ubiquitination level of $\mathrm{H} 2 \mathrm{~B}$ showed significant difference between RAD6B-deficient mouse brains and WT mouse brains, resulting in subsequent repair failures in neurons.

The developing and mature central nervous system responds differently to DNA damage, with the former tending to apoptosis, while the consequences of latter is less known (Lee and Mckinnon, 2007). Duchaud, E. et al. reported that mutations in ATM gene result in increased sensitivity of cells to ionizing radiation, however, they show less apoptosis than normal cells after irradiation (Roos and Kaina, 2006). Similarly, in this study, we noticed a remarkable increase in genomic instability in RAD6B deficient neurons, but no significant apoptosis compared with neurons from WT mice. Unrepaired DBSs caused by NHEJ deficiency have been reported to accelerate aging in mice and analysis of DSBR during aging revealed that cells from older mice contained more DSB than those from younger mice (Reynolds and Stewart, 2013; Rulten and Caldecott, 2013; Madabhushi et al., 2014). In addition, Approximately 20-40\% of the cerebral cortex and hippocampus region in the brains 
of older C57BL/6 mice has been observed to have severe DNA damage, heterochromatin formation, and senescence-associated $\beta$-galactosidase activation (Jurk et al., 2012). Jurk et al. (2012) reported that neurons develop a p21-dependent senescence-like phenotype mediated by failed DNA damage response. Western blot showed elevated expression of p53 and p21 which may stimulate subsequent responses and cause senescence. And slices from RAD6B-deficient mice exhibit more deeply stained nuclei and increased activation of astrocytes compared to those from WT mice, suggesting more unrepaired lesions in those neurons and more neurons undergoing neurodegeneration associated with aging. Further, the loss of neurons was more severe in RAD6B-deficient mice than in WT mice, which corresponded to the decrease in ubiquitination of $\mathrm{H} 2 \mathrm{~B}$ and an increase in p53 and p21 expression. In combination with these results, the absence of RAD6B was responsible for the failure of repair, leading to neuronal senescence, and neurodegeneration.

As the mice grew, we observed physical and behavioral differences between mice; the RAD6B-deficient mice were lighter in body weight than the WT mice and appeared less active than the WT mice. We further explored the changes in their learning and memory through behavioral experiments. RAD6Bdeficient mice showed reduced learning and memory ability in behavioral tests, indicating impairment in learning, and memory. In combination with neuronal changes in the mutant mice, we suggest that neurodegeneration may lead to these behavioral differences between RAD6B-deficient and wild-type mice.

Collectively, our data indicate that, in the central nervous system, RAD6B is essential for DDR and RAD6B deficiency causes defects in DNA DSBs repair and increased genome instability, which accelerates neuronal senescence and leads to neurodegeneration.

\section{MATERIALS AND METHODS}

\section{Generation of RAD6B KO Mice and X-Ray Irradiation}

RAD6B knockout C57BL/6 embryonic stem (ES) cells were generated by the knockout mouse project (KOMP) repository (UC Davis). The ES cells contained an allele [Ube2btm1 (KOMP) Vlcg] with an ablating deletion of the RAD6B gene, inserted in place of the WT allele by an expression-selection cassette (VelociGene KOMP definitive null allele design). The ES cells were implanted in the uteri of mice to produce transgenic mice. RAD6B knockout mice were obtained by crossing the mice with lines containing CMV-cre for systemic knockout and Nescre for neuron-conditional knockout (Supplementary Figures S1A,B). All mice were feed and bred in an SPF laboratory at the animal center of Lanzhou University. We randomly selected RAD6B-/-, mice and homologous WT mice and divided them into four groups [WT, WT IR (with X-ray irradiation treatment), RAD6B-/- and RAD6B-/- IR]; each group contained 30 mice. X-ray irradiation was performed with an X-ray biological irradiation system (X-RAD $225 \mathrm{Cx}$, Pxinc). All experiments involved in this study were approved by the ethics committee of Lanzhou University.

\section{Tissues Sampling and Frozen Sections}

Mice from each group were anesthetized with isoflurane, then perfused and fixed with $4 \%$ neutral formaldehyde. The brains were totally dissected and soaked in $20 \%$ and $30 \%$ sucrose solution for gradient dehydration. All the sections were cut at a thickness of $25 \mu \mathrm{m}$ on a freezing microtome.

\section{Cells Culture}

The immortalized mouse hippocampal neuronal RPE1 cells were purchased from ATCC and cultured in DMEM with high glucose supplemented with $10 \%$ fetal bovine serum (Gibco, Frederick, $\mathrm{MD}$, United States) at $37^{\circ} \mathrm{C}$ in a humidified incubator with $5 \%$ $\mathrm{CO}_{2}$ and $95 \%$ air. While for SA- $\beta-$ Gal staining and micronucleus test, cells were seeded into coverslip in Petri dishes at a density of $1 \times 10^{5}$ cells per $\mathrm{mL}$ and grown for days until $70-80 \%$ confluent.

\section{Silencing of RAD6B Gene}

RPE1 cells between passages 5 and 15 were infected with lentivirus particles (GeneChem Co., Shanghai, China) at a multiplicity of infection (MOI) of approximately 50 to generate siRNA against RAD6B (siRAD6B) or non -targeting siRNA (siNeg) [siRAD6B: 5' ACCAGAAGGGAC ACCCTTTGAAGATG3'; siNeg: negative control (GeneChem)]. $12 \mathrm{~h}$ after infection, the medium was replaced by complete medium, and cultured for subsequent days. The RNA interference efficiency was confirmed by western blot.

\section{Immunofluorescence Staining}

Brain slices from 6-month-old WT IR and RAD6B-/- IR mice were subjected to immunofluorescence staining as described by Liu et al. (2013). The primary antibodies used were anti- $\gamma-\mathrm{H} 2 \mathrm{AX}$ (ab26350, Abcam; 1:200), anti-GFAP (BA0056, BOSTER; 1:150), anti-BRCA1 (ab26350, Abcam; 1:200), anti-53BP1 (ab36823, Abcam; 1:200), anti-MDC1 (ab11169, Abcam; 1:200), and antiRNF8 (ab26350, Abcam; 1:200). After the nuclei were stained with DAPI, photomicrographs were captured with an olympus fluorescence microscope (BX53).

\section{Western Blot Assay}

Brain tissues from 6-month-old mice were frozen quickly in liquid nitrogen, after which histones, and total protein were extracted according to the standard methods provided by Abcam. The concentration of each protein of interest was measured with an ultra-microspectrophotometer (DS-11 FX, DeNovix). We performed automated western blots with Simple Western according to the manufacturer's instructions. And histones were detected with primary antibodies against H1.2 (19649, Proteintech; 1:500), ub-H2A (clone E6C5, Millipore; 1:500), H2A (ab36823, Abcam; 1:200), ub-H2B (clone 56, Millipore; 1:500), H2B (15857, Proteintech; 1:1000) and H4 (16047, Proteintech; $1: 2000)$, while total protein was reacted with antibodies against p21 (ab109199, Abcam; 1:1000), p16 (ab108349, Abcam; 1:1000), p53 (10442-1-AP, Proteintech; 1:500), AIF (17984-1-AP, Proteintech; 1:500), caspase-3 (19677-1-AP, Proteintech; 1:500), and GAPDH (10494-1-AP, Proteintech; 1:2000). 


\section{Cell Proliferation}

RPE1 cells were seeded at 1000 cells/well in 96-well dishes. After having been incubated for $12 \mathrm{~h}$, cells were irradiated at dose of $2 \mathrm{~Gy}$ or not. Following 24, 48, and $72 \mathrm{~h}$ of incubation, cell proliferation was measured by CCK- 8 assay.

\section{Micronucleus Test}

Following irradiation at a dose of $2 \mathrm{~Gy}$, cells were washed three times with phosphate buffer saline (PBS) and continued to culture in DMEM containing cytochalasin B at a concentration of $3 \mu \mathrm{g} / \mathrm{ml}$ for $16 \mathrm{~h}$ to prevent cell division. After incubation, cells were washed and fixed with $10 \%$ neutral formaldehyde for at least $30 \mathrm{~min}$. The cells were washed gently with PBS and stained with DAPI, and photomicrographs were captured with an BX53.

\section{Cresyl Violet (CV) Staining}

After X-ray irradiation at a dose of 10Gy and days of recovery, slices from 6-, 9-, and 12-month-old mice were stained with $0.5 \%$ $\mathrm{CV}$ for $15 \mathrm{~min}$ and differentiated in $0.25 \%$ glacial acetic acid ethanol solution for $15 \mathrm{~s}$, until the slices turned light blue. The slices were dehydrated with graded ethanol, cleared in xylene and covered with neutral balsam.

\section{Silver Staining}

Brain slices from 12-month-old mice were incubated in $20 \%$ silver nitrate for $30 \mathrm{~min}$ at $37^{\circ} \mathrm{C}$. After being washed 3 times in $0.01 \mathrm{M}$ PBS, they were reduced in $10 \%$ neutral formaldehyde for $3 \mathrm{~min}$, until they had turned brown. Following incubation in silver ammonia solution for $5 \mathrm{~min}$ at $37^{\circ} \mathrm{C}$, slices were placed in $10 \%$ neutral formaldehyde for $40 \mathrm{~s}$ and toned with $0.2 \%$ gold chloride solution. Finally, the slices were rinsed in 5\% sodium thiosulfate solution for $2 \mathrm{~min}$. After being washed adequately with distilled water, all slices were dehydrated and covered with neutral gum.

\section{SA- $\beta$-GAL Staining}

Senescent cells were stained with a Senescence $\beta$-Galactosidase Staining Kit (C0602, Beyotime) according to the standard procedures. The slices were fixed at room temperature for $20 \mathrm{~min}$. After being washed three times, each slice was stained with the dyeing solution and incubated at $37^{\circ} \mathrm{C}$ overnight. The positive cells were observed by microscope after routine dehydration and mounting. Detection of senescence-associated $\beta$-galactosidase in RPE1 cells was also performed following the instructions.

\section{Behavioral Tests}

RAD6B ncKO mice were subjected to the Morris water maze and a passive avoidance response test. For the Morris water maze, all mice were trained for 5 min on 7 consecutive days and assessed on the eighth day. The escape latency, total distance and time spent in the effective region were recorded automatically by a monitoring system (WMT-100, TECHMAN SOFT).

Passive avoidance response was performed with a video analysis system (PAT-8, TECHMAN SOFT). After the mice were allowed to move freely between the light chamber and the dark chamber for $3 \mathrm{~min}$, the metal grids of the dark chamber were electrified with a voltage of 24 volts for $5 \mathrm{~min}$. Prior to the formal test, all mice underwent two consecutive days of regular exposure and training. The Error counts and latency were recorded every $3 \mathrm{~s}$.

\section{DATA AVAILABILITY}

All datasets generated for this study are included in the manuscript and/or the Supplementary Files.

\section{ETHICS STATEMENT}

All experiments involved in this study were approved by the ethics committee of Lanzhou University.

\section{AUTHOR CONTRIBUTIONS}

ZG and DW initiated the project and wrote the manuscript. ZG, YS, YT, and YG designed the experiments. ZG, YS, YT, YG, and BL performed the experiments. XL, YT, YG, and BL analyzed the data. KX, YG, and DW constructed the figures.

\section{FUNDING}

This work was supported by the National Natural Science Foundation of China (Grant Nos. 81472541 and 81772907 to DW and No. 81571362 to YS) and the Fundamental Research Funds for the Central Universities (Grant lzujbky-2017-k12 to DW).

\section{SUPPLEMENTARY MATERIAL}

The Supplementary Material for this article can be found online at: https://www.frontiersin.org/articles/10.3389/fncel.2019. 00392/full\#supplementary-material

FIGURE S1 | Implementation of RAD6B-deficient mice. (A) Editing the RAD6B gene through the Cre-loxP recombination system. CMV-cre and Nes-cre were used to produce systemic knockout mice and ncKO mice, respectively. (B) Identification of the genotypes of some offspring mice obtained from RAD6B heterozygous mice. Primers were designed for genome amplification. (C) Physical comparison of 4-month-old WT mice and RAD6B ncKO mice. (D) The scatter diagram shows weight differences between 18-month-old WT mice and RAD6B ncKO mice, each group containing 8 mice. All values are presented as the mean \pm SEM $(n=8)$. Student's $t$-test.

FIGURE S2 | More neurodegeneration was observed in the brains of RAD6B-deficient mice. (A) Immunofluorescence was performed with anti-NeuN in the brain slices from RAD6B-deficient mice and control mice that were treated with 10Gy of X-rays or not. Scale bar $=20 \mu \mathrm{m}$. (B) The scatter diagram displays the relative density of neurons in the cerebral cortex of mice from each group. All values are presented as the mean \pm SEM $(n=18)$. Student's $t$-test. (C) Degeneration of nerve fibers (tangling, thickening, and staining) was observed in the silver-stained brain slices from RAD6B-deficient mice. Scale bar $=10 \mu \mathrm{m}$. (D) In lipofuscin staining of brain slices from RAD6B-deficient mice, microglia clustered around degenerated neurons. Scale bar $=10 \mu \mathrm{m}$. (E) The scatter diagram displays the proportion of abnormal neurons. All values are presented as the mean $\pm \operatorname{SEM}(n=18)$. Student's $t$-test. 


\section{REFERENCES}

Assenmacher, N., and Hopfner, K.-P. (2004). MRE11/RAD50/NBS1: complex activities. Chromosoma 113, 157-166. doi: 10.1007/s00412-004-030 6-304

Bartkova, J., Tommiska, J., Oplustilova, L., Aaltonen, K., Tamminen, A., Heikkinen, T., et al. (2008). Aberrations of the MRE11-RAD50-NBS1 DNA damage sensor complex in human breast cancer: mrell as a candidate familial cancer-predisposing gene. Mol. Oncol. 2, 296-316. doi: 10.1016/j.molonc.2008. 09.007

Barzilai, A. (2010). DNA damage, neuronal and glial cell death and neurodegeneration. Apoptosis 15, 1371-1381. doi: 10.1007/s10495-010-0501-0

Barzilai, A., and Mckinnon, P. J. (2013). Genome maintenance in the nervous system; insight into the role of the DNA damage response in brain development and disease. DNA Repair 12, 541-542. doi: 10.1016/j.dnarep.2013. 06.005

Biochem, A. R. (1998). The ubiquitin system. Annu. Rev. Biochem. 67, 425-479.

Braak, H., and Braak, E. (1998). Argyrophilic grain disease: frequency of occurrence in different age categories and neuropathological diagnostic criteria. J. Neural Transm. 105, 801-819. doi: 10.1007/s00702005 0096

Bunting, S. F., Callen, E., Wong, N., Chen, H. T., Polato, F., Gunn, A., et al. (2010). 53BP1 inhibits homologous recombination in Brcal-deficient cells by blocking resection of DNA breaks. Cell 141, 243-254. doi: 10.1016/j.cell.2010. 03.012

Campisi, J. (2005). Senescent cells, tumor suppression, and organismal aging: good citizens, bad neighbors. Cell 120, 513-533.

Canugovi, C., Misiak, M., Ferarelli, L. K., Croteau, D. L., and Bohr, V. A. (2013). The role of DNA repair in brain related disease pathology. DNA Repair 12, 578-587. doi: 10.1016/j.dnarep.2013.04.010

Cao, X., and Li, M. (2015). A new pathway for senescence regulation. Genomics Proteomics Bioinformatics 13, 333-335. doi: 10.1016/j.gpb.2015.11.002

Chen, S., Wang, D. L., Liu, Y., Zhao, L., and Sun, F. L. (2012). RAD6 regulates the dosage of $\mathrm{p} 53$ by a combination of transcriptional and posttranscriptional mechanisms. Mol. Cell. Biol. 32, 576-587. doi: 10.1128/MCB.059 66-11

Chernikova, S. B., Razorenova, O. V., Higgins, J. P., Sishc, B. J., Nicolau, M., Dorth, J. A., et al. (2012). Deficiency in mammalian histone H2B ubiquitin ligase Bre1 (Rnf20/Rnf40) leads to replication stress and chromosomal instability. Cancer Res. 72, 2111-2119. doi: 10.1158/0008-5472.CAN-11-2209

Ciechanover, A. (1994). The ubiquitin-proteasome proteolytic pathway. Cell 79, 13-21. doi: 10.1016/0092-8674(94)90396-4

Escribano-Díaz, C., Orthwein, A., Fradet-Turcotte, A., Xing, M., Young, J. F., Tkáè, J., et al. (2013). A cell cycle-dependent regulatory circuit composed of 53BP1RIF1 and BRCA1-CtIP controls DNA repair pathway choice. Mol. Cell 49, 872-883. doi: 10.1016/j.molcel.2013.01.001

Fradet-Turcotte, A., Canny, M. D., Escribano-Díaz, C., Orthwein, A., Leung, C. C. Y., Huang, H., et al. (2013). 53BP1 is a reader of the DNA-damageinduced H2A Lys 15 ubiquitin mark. Nature 499, 50-54. doi: 10.1038/nature1 2318

Game, J. C., and Chernikova, S. B. (2009). The role of RAD6 in recombinational repair, checkpoints and meiosis via histone modification. DNA Repair 8, 470482. doi: 10.1016/j.dnarep.2009.01.007

Gil, J., and Withers, D. J. (2016). Ageing: out with the old. Nature 530, 164-165. doi: 10.1038/nature16875

Harper, J. W., and Elledge, S. J. (2007). The DNA damage response: ten years after. Mol. Cell 28, 739-745. doi: 10.1016/j.molcel.2007.11.015

Hegde, M. L., Bohr, V. A., and Mitra, S. (2017). DNA damage responses in central nervous system and age-associated neurodegeneration. Mech. Ageing Dev. 161(Pt A), 1-3. doi: 10.1016/j.mad.2017.01.010

Herrup, K., Li, J., and Chen, J. (2013). The role of ATM and DNA damage in neurons: upstream and downstream connections. DNA Repair 12, 600-604. doi: 10.1016/j.dnarep.2013.04.012

Iyama, T. (2013). DNA repair mechanisms in dividing and non-dividing cells. DNA Repair 12, 620-636. doi: 10.1016/j.dnarep.2013.04.015

Jackson, S. P. (2005). Conserved modes of recruitment of ATM, ATR and DNAPKcs to sites of DNA damage. Nature 434, 605-611. doi: 10.1038/nature0 3442
Jackson, S. P., and Bartek, J. (2009). The DNA-damage response in human biology and disease. Nature 461, 1071-1078. doi: 10.1038/nature08467

Jeggo, P. A., Geuting, V., and Löbrich, M. (2011). The role of homologous recombination in radiation-induced double-strand break repair. Radiother. Oncol. 101, 7-12. doi: 10.1016/j.radonc.2011. 06.019

Jurk, D., Wang, C., Miwa, S., Maddick, M., Korolchuk, V., Tsolou, A., et al. (2012). Postmitotic neurons develop a p21-dependent senescence-like phenotype driven by a DNA damage response. Aging Cell 11, 996-1004. doi: 10.1111/j. 1474-9726.2012.00870.x

Kim, J., Guermah, M., McGinty, R. K., Lee, J. S., Tang, Z., Milne, T. A., et al. (2009). RAD6-Mediated transcription-coupled H2B ubiquitylation directly stimulates H3K4 methylation in human cells. Cell 137, 459-471. doi: 10.1016/j.cell.2009. 02.027

Kleiner, R. E., Verma, P., Molloy, K. R., Chait, B. T., and Kapoor, T. M. (2015). Chemical proteomics reveals a [gamma]H2AX-53BP1 interaction in the DNA damage response. Nat. Chem. Biol. 11, 807-814. doi: 10.1038/nchembio. 1908

Lavin, M. (2007). ATM and the Mrel1 complex combine to recognize and signal DNA double-strand breaks. Oncogene 26, 7749-7758. doi: 10.1038/sj.onc. 1210880

Lee, Y., and Mckinnon, P. J. (2007). Responding to DNA double strand breaks in the nervous system. Neuroscience 145, 1365-1374. doi: 10.1016/j.neuroscience.2006.07.026

Li, T., Kon, N., Jiang, L., Tan, M., Ludwig, T., Zhao, Y., et al. (2012). Tumor suppression in the absence of p53-mediated cell-cycle arrest, apoptosis, and senescence. Cell 149, 1269-1283. doi: 10.1016/j.cell.2012. 04.026

Liu, C., Wang, D., Wu, J., Keller, J., Ma, T., and Yu, X. (2013). RNF168 forms a functional complex with RAD6 during the DNA damage response. J. Cell Sci. 126(Pt 9), 2042-2051. doi: 10.1242/jcs.122945

Löbrich, M., Shibata, A., Beucher, A., Fisher, A., Ensminger, M., Goodarzi, A. A., et al. (2010). $\gamma \mathrm{H} 2 \mathrm{AX}$ foci analysis for monitoring DNA double-strand break repair: strengths, limitations and optimization. Cell Cycle 9, 662-669. doi: 10.4161/cc.9.4.10764

Madabhushi, R., Pan, L., and Tsai, L.-H. (2014). DNA damage and its links to neurodegeneration. Neuron 83, 266-282. doi: 10.1016/j.neuron.2014.06.034

Mandemaker, I. K., Van, C. L., Janssens, R. C., Lans, H., Bezstarosti, K., Hoeijmakers, J. H., et al. (2017). DNA damage-induced histone H1 ubiquitylation is mediated by HUWE1 and stimulates the RNF8RNF168 pathway. Sci. Rep. 7:15353. doi: 10.1038/s41598-017-15 194-y

Marteijn, J. A., Bekker-Jensen, S., Mailand, N., Lans, H., Schwertman, P., Gourdin, A. M., et al. (2009). Nucleotide excision repair-induced H2A ubiquitination is dependent on MDC1 and RNF8 and reveals a universal DNA damage response. J. Cell Biol. 186, 835-847. doi: 10.1083/jcb.20090 2150

Ng, H. H., Xu, R. M., Zhang, Y., and Struhl, K. (2002). Ubiquitination of histone H2B by Rad6 is required for efficient dot1-mediated methylation of histone H3 lysine 79. J. Biol. Chem. 277, 34655-34657. doi: 10.1074/jbc.c20043 3200

Ouyang, S., Song, Y., Tian, Y., Chen, Y., Yu, X., and Wang, D. (2015). RNF8 deficiency results in neurodegeneration in mice. Neurobiol. Aging 36, 2850 2860. doi: 10.1016/j.neurobiolaging.2015.07.010

Reynolds, J. J., and Stewart, G. S. (2013). A nervous predisposition to unrepaired DNA double strand breaks. DNA Repair 12, 588-599. doi: 10.1016/j.dnarep. 2013.04.011

Roos, W. P., and Kaina, B. (2006). DNA damage-induced cell death by apoptosis. Trends Mol. Med. 12, 440-450. doi: 10.1016/j.molmed.2006.07.007

Rulten, S. L., and Caldecott, K. W. (2013). DNA strand break repair and neurodegeneration. DNA Repair 12, 558-567. doi: 10.1016/j.dnarep.2013.04. 008

Sherr, C. J., and McCormick, F. (2002). The RB and p53 pathways in cancer. Cancer Cell 2, 103-112. doi: 10.1016/s1535-6108(02)00102-2

Thorslund, T., Ripplinger, A., Hoffmann, S., Wild, T., Uckelmann, M., Villumsen, B., et al. (2015). Histone H1 couples initiation and amplification of ubiquitin signalling after DNA damage. Nature 527, 389-393. doi: 10.1038/nature1 5401 
Van Oss, S. B., Shirra, M. K., Bataille, A. R., Wier, A. D., Yen, K., Vinayachandran, V., et al. (2016). The histone modification domain of Pafl complex subunit Rtf1 directly stimulates H2B ubiquitylation through an interaction with Rad6. Mol. Cell 64, 815-825. doi: 10.1016/j.molcel.2016.10.008

Varshavsky, A. (2005). Regulated protein degradation. Trends Biochem. Sci. 30, 283-286.

Walden, H., Podgorski, M. S., and Schulman, B. A. (2003). Insights into the ubiquitin transfer cascade from the structure of the activating enzyme for NEDD8. Nature 422, 330-332.

Wang, D., Yu, T., Liu, Y., Yan, J., Guo, Y., Jing, Y., et al. (2016). DNA damage preceding dopamine neuron degeneration in A53T human alpha-synuclein transgenic mice. Biochem. Biophys. Res. Commun. 481, 104-110. doi: 10.1016/j. bbrc.2016.11.008

White, R. R., and Vijg, J. (2016). Do DNA double-strand breaks drive aging? Mol. Cell 63, 729-738. doi: 10.1016/j.molcel.2016.08.004
Yoshida, K., Hata, Y., Kinoshita, K., and Nishida, N. (2017). Argyrophilic grain disease in a 46-year-old male suicide victim. J. Neurol. Sci. 380, 223-225. doi: 10.1016/j.jns.2017.07.043

Conflict of Interest Statement: The authors declare that the research was conducted in the absence of any commercial or financial relationships that could be construed as a potential conflict of interest.

Copyright (C) 2019 Guo, Tian, Guo, Li, Liu, Xie, Song and Wang. This is an openaccess article distributed under the terms of the Creative Commons Attribution License (CC BY). The use, distribution or reproduction in other forums is permitted, provided the original author(s) and the copyright owner(s) are credited and that the original publication in this journal is cited, in accordance with accepted academic practice. No use, distribution or reproduction is permitted which does not comply with these terms. 